\title{
Study on Comparative Prevalence of Lungworms of Sheep and Cattle Slaughtered at Addis Ababa Abattoir, Ethiopia
}

\author{
Zarihun Wolde $^{1}$, Tesfaye Mersha ${ }^{2}$ \\ ${ }^{1}$ Animal Health supervisor at Weliso district Bureau of Livestock and Fishery development, South \\ West Shewa, \\ ${ }^{2}$ Researcher at Oromia Agricultural Research Institute (OARI), Bako Agricultural Research Center \\ (BARC), Ethiopia
}

\begin{abstract}
Study was conducted in Addis Ababa municipal abattoir from November 2014 to March 2015 with the objectives of determining prevalence comparison of lungworm infection in sheep and cattle, identifying the species of the respiratory helminthes circulating in the area and assessing possible risk factors of lung worm infection in sheep and cattle in the study area. Post mortem examination had been done on 400 sheep and 1000 cattle in Addis Ababa municipal abattoir. The overall prevalence of lung worm infection in post mortem finding was $49.5 \%$ and $1.5 \%$ in sheep and cattle, respectively. The potential risk factors to be considered were age, body condition, and species of lung worm have significant difference $(p<0.05)$ on prevalence of sheep and cattle's lung worm infection. Prevalence of young and adult age groups of sheep was67.33\% and $43.48 \%$, respectively as post mortem finding confirmed as well as theprevalence of young and adult age groups of cattle's were $5.19 \%$ and $0.83 \%$, respectively according to the post mortem finding result revealed. As well as Prevalence of sheep lung worms based on their body conditions (poor, medium and good) were 63.08\%, 3.76\% and $47.52 \%$, respectively and prevalence of Cattle lungworms based on their body conditions (poor, medium and good) were $0.97 \%, 37.98$, and $0.8 \%$, respectively.The prevalence of D. filarial (23\%) was the highest and D.vivparus (1.50\%) and mixed infection (3.5\%) were the lowest whereas prevalence of M. capillaries $(14.75 \%)$ and P. rufescens( $8.25 \%)$ were moderateas the result of post mortem finding of this study showed.Therefore, due to its great impact on production of sheep and cattle in general as a country, emphasis should be given to control and prevention of lungworm infection and further study should be conducted in future to identify the temporal pattern of this parasitic disease.
\end{abstract}

Keywords:sheep, cattle, lungworm, Municipal-abattoir,prevalence,risk factors, Addis Ababa.

\section{INTRODUCTION}

Parasites pose subtle economic loss and are the major factors responsible for lowered level of production in tropical and subtropical regions particularly helminthes infection are among the most prevalent and widely distributed ones. ${ }^{[1]}$ Infections with gastrointestinal and other helminthes parasites are among the most neglected areas of veterinary care in much of the developing world. However, it has been established that high prevalence of the infection with less obvious signs associated with poor production and unthriftness. ${ }^{[2]}$ Among many contrasts which limit productivity in livestock populations helminthes parasites particularly lung worms of ruminants are of major importance. The species of importance in ruminants belongs to two different families(dictycaulidae and metastrongylidae).The dictycaulidae includes dictycaulus viviparous in cattle and buffaloes anddictycaulus filarial in sheep and goats. These worms are 5-10cm long and live in the trachea and bronchi. The metastorngylidiae are represented by at least three species in small ruminants. Protostlongylusrufisence a small worm $(1.5-3.5 \mathrm{~cm})$ found in bronchioles, mulleries capillaries (1.2$2.5 \mathrm{~cm})$ which are located in the alveoli and cystocaulsocreatus $(2-5 \mathrm{~cm})$ found in the terminal bronchioles ${ }^{[2]}$.

Lung worms are widely distributed throughout the world providing nearly perfect conditions for their survival and development but are particularly common in countries with temperate climates, and in the highlands of tropical and sub-tropical countries. Dictyocaulidae and/or certain Metastrongylide are known to exist in East Africa (Ethiopia, Kenya, and Tanzania) and South Africa. ${ }^{[2]}$ Control of these parasites is, therefore, essential for releasing the potential of small ruminant production. For proper 
control to be instituted, however, diseases and their dynamics must be known. At our present state of knowledge of parasitic diseases, it is difficult and even dangerous to lay down rigid rules for their control which are applicable to all regions. For this reason a study of epidemiology of each parasitic disease should be limited to small areas. ${ }^{[3]}$ The incidence of parasitic diseases, including respiratory helminthosis varies greatly from place to place depending on the relative importance of many ofthe factors. In this regard, very few and limited studies were done so far pertaining to respiratory helminthes of sheep and cattle in Addis Ababa abattoir. Therefore, this study was designed with objectives of:

- To determine prevalence of lung worm in sheep and cattle and compare the prevalence among sheep and cattle,

- To identifying the species lung worms helminthes that circulating in the area and,

- To assessing the possible risk factors of lung worm infection in sheep and cattle in the study area.

\section{MAterials AND Methods}

\subsection{Study Area}

The study was conduct at Addis Ababa municipal Abattoir. The study abattoir is found in Addis Ababa city which is a capital city of Ethiopia and located in the heart of the country between 09002 $\mathrm{N}$ latitude and 380 44' E longitude and surrounded by Oromia regional state. It is stimulated in the altitude range between $2200-2500 \mathrm{~m}$ above sea level. The city lies at the foot of the 3000, high Enteto Mountain and it covers a total area of 540sqk. Despite its proximity to the equator, Addis Ababa enjoys a mild, afro alpine temperate and warm temperature. The rain fall is bimodal with highest rain fall occurs in July and fall is $1188.2 \mathrm{ma} .{ }^{[4]}$ The mean annual temperature varies from $120 \mathrm{c}-240 \mathrm{c}$. The hottest months are May and June while the coldest months are October and November. Addis Ababa abattoir was established by the emperor of Hailesilasie in 1956 and nowadays, they enterprise consists of 800 permanent workers on average.

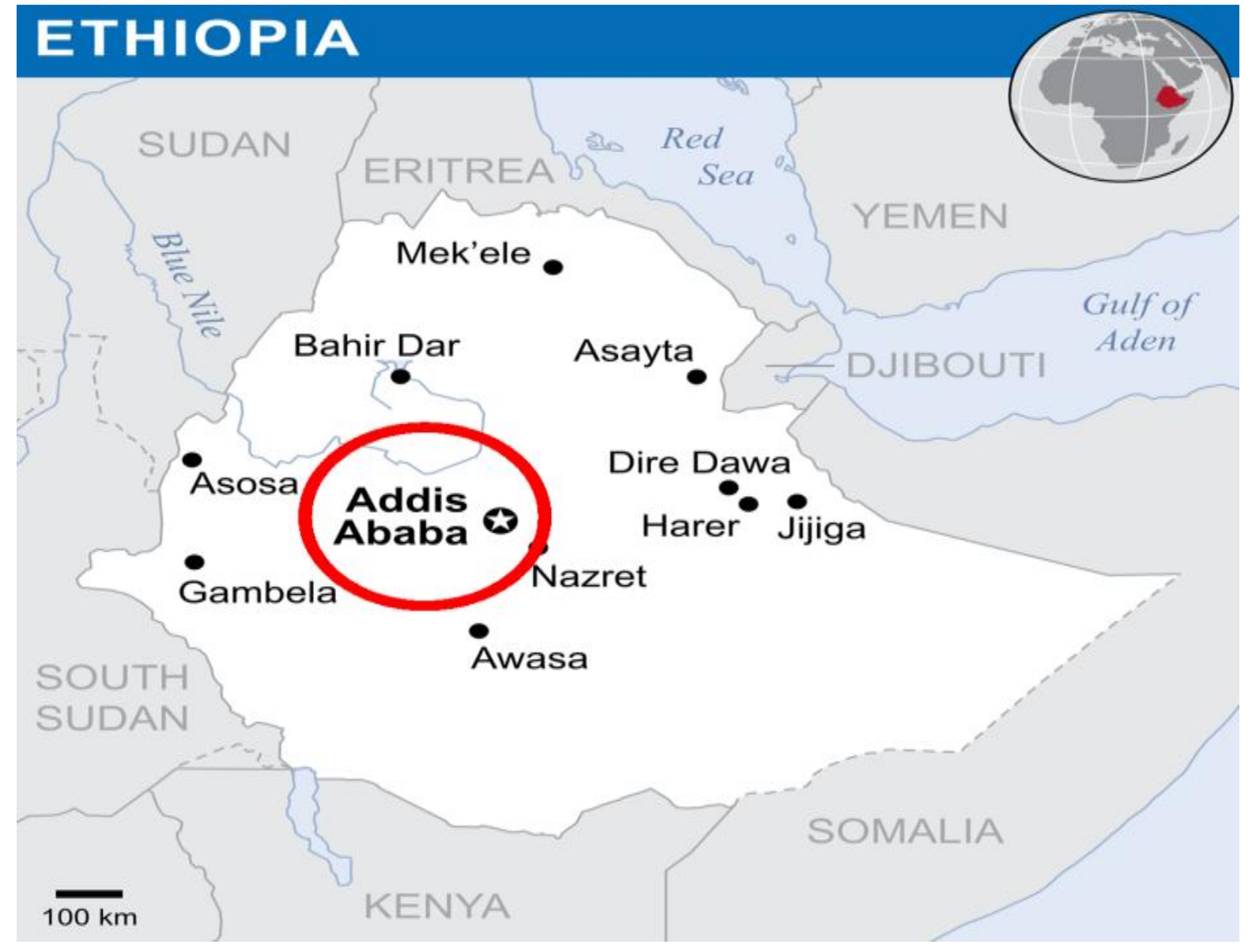

Fig1. Shows map of the study area 


\subsection{Study Population}

A number of cattle and sheep were presented to the slaughter house from different parts of the country for slaughteringpurpose in each day. However, Borena, Harar, central and northern Shewa, Wolayitasodo, Bale, Jimma and surrounding areas are the major source of animals. Usually the number of animals slaughtered daily is not uniform, and around 600 cattle are slaughtered per day on average and the number may increase up to 1000 on Wednesday and Friday. An investigation was carried out on these animals five times per week taking 50 bovine and 20 ovine on each day by random selection with special emphasis on animals having respiratory signs.

\subsection{Sample Size Determination}

A sample of an individual was selected from a larger population of livestock areas of the country for slaughtering purpose, then determination of the presence or absences of disease carried out simultaneously for each individual and eventually hypothesize risk factors. The study was crosssectional, with simple random sampling techniques, the desired sample size was calculated the formula derived by ${ }^{[5]}$ with $95 \%$ confidence interval $(\mathrm{CL})$, and at $5 \%$ absolute precision and with assumption of $50 \%$ expected prevalence of ovine lung worm. The sample size of both bovine and ovine were determined by considering prevalence of $0.5 \%$ which recorded by ${ }^{[6]}$ in Addis Ababa abattoir previously and the sample size of 764 and 384 cattle and sheep were considered for the study, respectively. However, 1000 cattle and 400 sheep ware examined for post mortem examination in order to increase the precision.

$$
\mathrm{n}=\frac{\text { 1.962. Pexp (1-Pexp) }}{\mathrm{d}^{2}}
$$

Where; $\mathrm{n}=$ required sample size

Pex $=$ expected prevalence

$\mathrm{d}=$ desired absolute precision

$1.96=\mathrm{z}$ value at $95 \%$ confidence interval with $5 \%$ absolute precision

If pex $=0.005$ and $d=0.05$ then $n=764$ for cattle

\subsection{Study Methodology and Sample Collection}

\subsubsection{Post Mortem Examination}

To study the prevalence of bovine and ovine lung worm, post mortem examination through inspection, palpation and incision of lung was made and the rate of infection of lung worm were recorded. About 1000 and 400 of bovine and ovine samples were taken randomly from the animals slaughtered in Addis Ababa abattoir. A systematic (five slaughtering days/week) visit was made at Addis Ababa municipal abattoir from November 2014-march 2015. During ante mortem inspection each of the study animals were given an identification number cultish a paint mark on their body). Age, sex and body condition scoring of the study animals was also recorded. The air passages were opened starting from the trachea down to the bronchi with fine blunt pointed scissors to detect parasites.

Estimation of age was done by the examination of teeth eruption using the approach forwarded by ${ }^{[7]}$.Two age groups were considered; less or equal to five years and above five years. Since almost all the cattle presented to slaughtering in the study area were male, infection rate regarding sex variation was not included. The body condition scoring was classified in to three categories as lean $(1,2,3)$, medium $(4,5,6)$ and fat $(7,8,9)$.

\subsection{Statistical Analysis}

The data were entered and managed in MS excel work sheet programmer was employed to create data base and SPSS statistical software version 16.0 was used to analyze the data. The prevalence of lungworm infection was calculate for different risk factors by dividing the total number of positive animals to the total number of examined animals, which observed at that particular time .Chi square statistics were used to compare the association between variables and descriptive statistics to shown the data in (Tables). 


\section{ZarihunWolde \&TesfayeMersha}

The significance of differences between the prevalence of lungworm species were determined using fisher's exact test, when the number with in the categories was too small for Chi-square test. The explanatory variables were (species of examination, age, body condition) considered as risk factors to see their association with the level of prevalence.

\section{RESUltS}

\subsection{Lungworm Infection inCattle}

Out of the 1000 cattle, $150(1.5 \%)$ of them found to be harboring D. Viviparous in their lungs, the prevalence of lungworm infection showed significance different $(\mathrm{p}<0.05)$ between the two age groups (Table 1).

Table1.Prevalence of cattle lungworm in different age groups

\begin{tabular}{|l|l|l|l|}
\hline Age & No. animal examined & No. Positive & 95\% CI \\
\hline$\leq 5$ years & 154 & $8(5.2)$ & $2.3-8.2$ \\
\hline$>5$ years & 846 & $7(0.83)$ & $0.3-1.3$ \\
\hline Total & 1000 & $15(1.5)$ & $0.3-2.1$ \\
\hline
\end{tabular}

$X^{2}=16.81, p=0.000$

The prevalence of lungworm infection in cattle with different body conditions (poor, medium and good ) was found to be high in animals of body condition (Table 2), when compared with those of medium and good body conditions $(\mathrm{p}<0.05)$. The species of lungworm involved in cattle in all positive animals $D$. Viviparous

Table2.Prevalence of cattle lungworm infection in different body conditions

\begin{tabular}{|l|l|l|l|}
\hline & No. examined & No. Positive animals (\%) & Type of body95\% CI \\
\hline Poor & 212 & $8(3.8)$ & $1.6-5.9$ \\
\hline Medium & 412 & $4(0.97)$ & $0.2-1.8$ \\
\hline Good & 376 & $3(0.79)$ & $0.04-1.56$ \\
\hline Total & 1000 & $15(1.5)$ & $0.3-2.1$ \\
\hline
\end{tabular}

$X^{2}=9.43, p=0.009$

\subsection{Lungworm Infection in Sheep}

The overall lungworm prevalence of sheep recorded in this study was $49.5 \%(n=198)$. The species of lungworm involved in this study were d. Filarial, m, capillaries and P. rufecens with prevalence of $23 \%, 14.8 \%$ and $8.3 \%$ respectively in decreasing order. Mixed infection was observed in $14(3.5 \%)$ of positive animals. An attempt was made to see the prevalence difference between two age groups (young and adults), the study reveal a significant variation $(\mathrm{p}<0.05)$ in the prevalence of lung worm infection among the two age groups. The prevalence being higher in young animals than adults * (Table 3)

Table3.Prevalence of ovine lung worm in different age groups

\begin{tabular}{|l|l|l|l|}
\hline Age & No. Animal Examined & No. Positive Animals & $(\%) 95 \%$ CI \\
\hline Young & 101 & $68(67.3)$ & $59.7-75.0$ \\
\hline Adult & 299 & $130(43.4)$ & $38.8-48.2$ \\
\hline Total & 400 & $198(49.5)$ & $45.4-53.6$ \\
\hline
\end{tabular}

$X^{2}=17.17, p=0.000$

Sheep with different body condition showed statistically significant variation for lungworm infection the prevalence being very high in sheep having poor body condition followed by medium and good body conditions (table 4)

Table4.Prevalence of ovine lungworm infection in different body conditions

\begin{tabular}{|l|l|l|l|}
\hline Body conditions & No. Examined & No.Positive & $(\%) 95 \%$ CI \\
\hline Poor & 130 & $82(63.1)$ & $56.1-70.0$ \\
\hline Medium & 141 & $67(47.5)$ & $40.6-54.4$ \\
\hline Good & 129 & $47(36.4)$ & $30.9-45$ \\
\hline Table & $\mathbf{4 0 0}$ & $\mathbf{1 9 8}(\mathbf{4 9 . 5})$ & $\mathbf{4 5 . 4 - 5 3 . 6}$ \\
\hline
\end{tabular}

$X^{2}=16.65, p=0.000$ 


\section{DISCUSSION}

The Overall prevalence of cattle lungworm infection (1.5\%) found in this study was very low this finding couldn't reflect the general situation because cattle slaughtered in Addis Ababa abattoir purchased from different market places of the country mostly from low $\mathrm{i}$ and areas because the climate altitude and rainfall are known to influence the abidance of lung worm ${ }^{[8][9]}$ in addition a significant number of cattle brought for slaughter in Addis Ababa abattoir come from feedlots. The probability of deworming may be high similar low prevalence of cattle lungworm infection has been reported by ${ }^{[6]}$ who reported $0.5 \%$ inAddis Ababa abattoir ${ }^{[9]}$ reported $1.1 \%$ in Malaysia on the contrary ${ }^{[10]}$ reported zero prevalence in the Kirikkale province of turkey. The overall prevalence $(49.05 \%)$ of sheep lungworm infection recorded in the presented work was high and it is comparable with findings of ${ }^{[1]}$ in and around Assella. ${ }^{[12]}$ Around Dessie, Kombolcha ${ }^{[13]}$ andChilallo area which reported prevalences of $60.5 \%, 67.83 \%, 81 \%$, respectively which are higher prevalence than the present study. But, it differs with those reports of ${ }^{[14]}$ in and aroundMekelle with prevalence of $33 \%{ }^{[15]}$ in and around Assella reported $39.4 \%{ }^{[16]}$ in and around Bahirdar with a report of $28.78 \%$ and ${ }^{[17]}$ in Gondar with prevalence rate of $31.74 \%$ such variation of infection rate could be attributed to the difference in study type sample size and study area.

The result of this study in sheep revealed that D. filaria was the most prevalent (23\%) of the total positive samples examined.This result agrees with the previous report of ${ }^{[17]}$ in Gonder town ${ }^{[13]}$ in Chilalo are ${ }^{[18]}$ around Debreberhan and ${ }^{[19]}$ in Hamsenaweraja but disagree with the report of ${ }^{[20]}$ in Bahirdar and ${ }^{[21]}$ in Addis Ababa who reported M. Capillaris to be the most prevalent lungworm species in their studies. This variation may be associated with the variation in the life cycle of the variation in the life cycle of the lungworm species for instance $D$. filariahas a direct life cycle and takes less time to reach the infective stage and after ingestion larvae can appear in feces within 5 week ${ }^{[22]}$, in addition transmission of $p$. rufescens and M. Capillarisis epidemiologically complex event involving host parasite and intermediate host in addition to this low prevalence of both $M$. Capillarisand. p. rufescens in the areas because of sampling was done during dry season which is not suitable to the snails inhabitance of these species to which larvae (L) develop to infective stage (L3) . $P$. rufescenswhose intermediate host range is restricted to certain species of snail has lower prevalence of the rest through its geographic rage is just wide like. In case of cattle the result of the present study showed that high prevalence of lungworm infection in animals of $<5$ years $(5.2 \%)$ when compared to animals of $>5$ years old $(0.83 \%)(\mathrm{p}<0.05)$. Similar report has been documented by ${ }^{[9]}$ and ${ }^{[23]}$ in Rwanda and USA, respectively this could be partly explained by the acquired immunity developed in older animals due to previous exposure and those of recovered animals from the infection have better immunity against reinfection. ${ }^{[3][24][25][26]}$

In analysis of the prevalence of lungworm infection in young and adult age groups of sheep also showed similar statistical significant difference among young and adult animals $(p<0.05)$. This finding is coincides with the previous study results of $\left.{ }^{[19][11][12][24}\right]$ similar reasons sated for cattle as to why younger animals are more affected as well as holds true for sheep. While assessing the influence of body condition score on the prevalence of lungworm infection in cattle and sheep animals with poor body conditions the prevalence of lungworm infection was found to high when compared to with animals of medium and good body conditions $(\mathrm{p}<0.05)$ even if there are no available similar research done in cattle to comparative to my findings. However the effect of body condition of study animals in the Prevalence of ovine lungworm infection was studied by the following scholars. The result of the present study in sheep agrees with the previous report of ${ }^{[27]}$ who reported prevalence of $64.5 \%$ $25.13 \%$ and $18 \%$ by in poor medium and good body condition respectively in and around Tseadaemba; ${ }^{[28]}$ also reported prevalence of $79.1 \% 52.1 \%$ and $30.9 \%$ in poor medium and good body condition respectively in Assela area the possible reason could be due to immune suppression in poor body condition concurrent infection by other parasites and GIT helminthes simultaneously as reported by ${ }^{[29]}$ and $^{[30]}$ on top of that poorly nourished sheep appear to be less competent in getting rid of lungworm infection. ${ }^{[31]}$

\section{CONCLUSION AND RECOMMENDATIONS}

The result of current study revealed that, lungworm is one of the major parasites of sheep and cattle in Addis Ababa abattoir. The lungworm infection rate dominantly existed in sheep and cattle with poor body condition and young age groups. Over all in the present study it was found that the prevalence in 
sheep $(49.5 \%)$ was higher than in cattle $(1.5 \%)$.one of the probable reasons may be due to the fact that for most of the sheep brought for slaughter from rural areas that graze in a free range, the probability of deworming may low and the presence of the parasite in the lung was high.

D. filaria is the most dominant ovine lungworm species, than M. capillaries and P. rufescens, whereas with mixed infection has the lowest prevalence. one of the probable reason for this is D. filaria has a direct life cycle and takes less time to reach the infective stage and after ingestion, larvae can appear in feces within 5 weeks.

Compared with the $D$. filaria, transmission of $P$. rufescens and $M$. capillaries are epidemiologically complex event involving host, parasite and intermediate host. In addition to this low prevalence of both $M$. capillaries and $P$. rufescensin the areas because of sampling was done during dry season which is not suitable to the snails, IH of these species to which larvae (L) develop to infective stage (L3).

Based on the present finding, the following recommendations are forwarded:-

Prohibition of sheep and cattle from grazing early in the morning and evening, and in swampy areas to protect them from infection.

* Treating of sheep of cattle with broad-spectrum anthelmintic at the beginning of rainy season could appear to be most effective.

* Isolation of most susceptible age groups during the season when pasture contamination occurs.

* Due to its impact on production, emphasis should be given for the control and prevention of lungworm infection in highland areas.

\section{REFERENCES}

[1] Bager I.A,(1998):Helminth parasites and animal production.in:LE.Symons,A,Donald and J.K.Dineen(eds).Biology and control of endo- parasites.Academic press, 133-135

[2] Hansen, J., Perry, B., (1994): The Epidemiology, Diagnosis and Control of Helminth Parasites of Ruminants. ILRAD, Nairobi, Kenya; Pp. 83.

[3] Radostits, O.M., Blood, D.C and Gay. C.C., (1994): Veterinary Medicine. A text book of the disease of Cattle, Sheep, Pigs, Goats, and Horses, 10th ed. Bailliere, Tindall, England, Pp. 15641568

[4] Addis Ababa administration city municipality (2005).

[5] Thrush Field, M. (2005): Department Vet. Clinical Studies Royal (Disk) School of Vet. Studies, University of Ediplug, 2nd, ed London.

[6] Fekadu, S., (2008): A study on Common Gross lungs of cattle slaughtered at Addis Ababa. Abattoirs DVM Thesis, Jimma University School of Veterinary Medicine, Jimma Ethiopia.

[7] De-Lahunta, a. and Habel, R*E (1986):Teeth, applied veterinary anatomy. W.B. Saunders Company Pp 4-6.

[8] Lat-Lat, H., Hassan, L., Sani, R.A., Sheikh-Omar, A.R.,Hishamfariz, M. \& Ng, V.(2007): First report of bovine lungworm disease in South-East Asia. Tropical Biomedicine 24(1): 77-81.

[9] Nishimiyimana,J.,Shyaka,A.andMutandwa,E.(2010):Effect of altitude and animal age on the prevalence of dictyocaulosis in cattle in the northen province of Rwanda,journal of agricultural extension and rural development,Vol.2(2)Pp.36-39.

[10] Kader,Y. (2006): Prevalence of lungworm infection in sheep and cattle in the kirikkaleprovince,turkiyeparazitoloji Dergisi,30(3):190-193.

[11] Wodowossen, T. (1992): Prevalence of lungworms in and around Assela. DVM Thesis, Faculty of Vet. Med. Addis Ababa University, Debre-Zeit, Ethiopia, Pp. 47.

[12] Tefera, S. (1993): Prevalence of lungworms around Dessie and Kombolcha, DVM Thesis, Faculty of Vet. Med. Addis Ababa University, Debre-Zeit, Ethiopia, Pp. 65.

[13] Paulos, A., (2000): Importance of seasonal dynamics of lungworms infection of small ruminant's in Chilalo areas, Arsi Zone. DVM Thesis, Addis Ababa University, Debre-Zeit, Ethiopian, Pp. $15-22$. 
[14] Frewengel,S (1995): Prevalence of ovine dictyocaulus in and around mekelle .DVM Thesis,AAU,FVM,Debrezeit, Ethiopia.

[15] Eyob, E. (2008): Prevalence of ovine lungworm infection in and around Assela. DVM Thesis, Jimma University School of Veterinary Medicine, Jimma Ethiopia.

[16] Muluken, Y., (2009): Prevalence lungworm infection in and around BahirdarGonder Town. DVM Thesis, Jimma University School of Veterinary Medicine, Jimma Ethiopia.

[17] Yohannes, E., (2010): Prevalence lungworm infection in small ruminants in Gonder Town. . DVM Thesis, Jimma University School of Veterinary Medicine, Jimma Ethiopia.

[18] Natsanet, B., (1992): Study on prevalence and control of lungworm in local Ethiopian highland sheep in and around DebreBrehan, Debre-Zeit, DVM Thesis. Faculty of Veterinary Medicine, Addis Ababa University, Debre-Zeit, Ethiopia, Pp. 42.

[19] Uqbazghi, K. (1990): Preliminary survey on the prevalence of lungworm in small ruminants HamassinAwraja. DVM Thesis, Faculty of Vet. Med. Addis Ababa University, Debre-Zeit, Ethiopia. Pp. 58.

[20] Sissay, A., (1996): Preliminary study on the prevalence of ovine lungworm infection in and around Bahirder. DVM Thesis, AAU-FVM, Debre-Zeit, Ethiopia, Pp. 69.

[21] Mezgebu, M., (1995): A survey on ovine fascioliasis and lungworm infection in Addis Ababa and the surrounding highland areas. DVM Thesis, AAU-FVM, Debre-Zeit, Ethiopia.

[22] Soulby, E.J.L. (1982): Helminthes, Arthropods and Protozoa of Domestic Animals, 6th ed., Bailliare, Tindall, London, Pp 499-552.

[23] Williams,J.C. and loyacano,A.F.(2001):Internal parasite of cattle in lousiania and other southern states,Lousiania agricultural experiment station,Lousianauniversity,agriculturalcenter,Balton Rouge.

[24] Abebe,M. (2008): Prevalence of lungworm infection in and around Assela, DVM Thesis, Mekale University, Faculty of Veterinary Science, Mekale Ethiopia.

[25] Urquhart, G.M., Armour, J., Duncan, J.L., Jenning, F.W., (1996): Vet.Parasitology. Longman English language Society, Pp. 39-58.

[26] Wilson, G., J. (1976): The strength and duration of D. filaria infection in sheep and goat Rest. Vet. Sci. Vol. 11(1), Pp. 7-17.

[27] Dawit, M., (2009): Prevalence of ovine lungworm in and around Tse-Ada-Emba. DVM Thesis, Jimma University School of Veterinary Medicine, Jimma Ethiopia.

[28] Hassen, A. (2009): Prevalence of ovine lungworm in and around Assela. DVM Thesis, Jimma University School of Veterinary Medicine, Jimma Ethiopia.

[29] Thomson, E.F., and Orita, G. (1988): Seasonal prevalence of Protostrongylid and Dictyocaulus species of Lungworms in Awassi Sheep in North-west Syria. Trop. Health Prod. 20, 187-189.

[30] Jovanovic, M., (1977): Report to the Ministry of Agriculture, Addis Ababa, Ethiopia.

[31] Kimberling, C.V., (1998): Jense and Smith Diseases of Sheep, 3rd ed. Lea and Febiger, Philadelphia, Pp. 99-101.

[32] Cringoli, G., Rinaldi, L., Veneziano, V., Capelli, G. and Malone, J. B. (2002). A cross sectional corpological survey from an area of southern Italian Apennines. Veterinary Parasitological 108 (2): 137-143.

[33] Nicholson, Mis., and Butter Worth, M.H. (1986): A Guide to Body condition scoring of Zebu cattle. International livestock center for Africa, Addis Ababa, Ethiopia. 